\title{
Combined factor VII/protein C deficiency results in intrauterine coagulopathy in mice
}

\author{
Joyce C.Y. Chan, ${ }^{1}$ Ivo Cornelissen, ${ }^{1}$ Desire Collen, ${ }^{2}$ Victoria A. Ploplis, ${ }^{1}$ \\ and Francis J. Castellino ${ }^{1}$ \\ ${ }^{1}$ W.M. Keck Center for Transgene Research and the Department of Chemistry and Biochemistry,
University of Notre Dame, Notre Dame, Indiana 46556, USA
${ }^{2}$ Center for Transgene Technology and Gene Therapy, Flanders Interuniversity Institute for Biotechnology,
B3000 Leuven, Belgium,
}

Address correspondence to: Francis J. Castellino, W.M. Keck Center for Transgene Research and the Department of Chemistry and Biochemistry, 229 Nieuwland Science Hall, University of Notre Dame, Notre Dame, Indiana, 46556 USA.

Phone: (219) 631-6456; Fax: (219) 631-8149; E-mail: castellino.1@nd.edu.

Received for publication December 8, 1999, and accepted in revised form February 2, 2000.

To determine whether an additional loss of the coagulation factor VII (FVII) gene influenced the coagulopathy observed in protein $\mathrm{C}$ gene-deficient $\left(\mathrm{PC}^{--}\right)$embryos and neonates, we crossed mice doubly heterozygous for the factor VII $\left(\mathrm{FVII}^{+-}\right)$and protein $\mathrm{C}\left(\mathrm{PC}^{+/}\right)$genes to produce offspring possessing the 9 predicted genotypic combinations. $\mathrm{FVII}^{-/-} / \mathrm{PC}^{-/-}$embryos, although present at their expected Mendelian frequency, displayed a phenotype that had not been observed in either the FVII or PC singly deficient embryos. At E12.5 days postcoitum (dpc), $F V I I^{-/-} / \mathrm{PC}^{-/-}$embryos demonstrated an intra- and extravascular coagulopathy that progressed with substantial concomitant hemorrhage and peripheral edema by E17.5dpc, resulting in mortality immediately after birth. $\mathrm{FVII}^{+/-} / \mathrm{PC}^{-/-}$embryos showed a less severe phenotype, suggesting a gene dosage effect. The lack of rescue of $\mathrm{PC}^{-/-}$embryos and neonates and augmented coagulopathy resulting from an additional heterozygous or homozygous FVII deficiency are probably due to increased factor $\mathrm{Xa}$ and thrombin generation, resulting from loss of FVIIa-dependent tissue factor pathway inhibitor function and the absence of control at the levels of factors Va and VIIIa. The presence of fibrin in embryos in the absence of fetal FVII suggests that significant clot-generating potential exists outside of the embryonic factor VII-dependent pathway.

J. Clin. Invest. 105:897-903 (2000).

\section{Introduction}

The process of blood coagulation is initiated and maintained by a systematic sequence of proteolytic activations of plasma zymogens that ultimately result in fibrin formation. Specifically, after vascular injury, tissue factor (TF), a membrane-bound protein that is normally sequestered from blood, is exposed to the plasma protein known as activated FVII (FVIIa). The resulting FVIIa/TF complex catalyzes the conversion of FIX and FX in plasma to the serine proteases FIXa and FXa, respectively. This leads to thrombin formation from prothrombin, and then to fibrin deposition at the site of vascular injury. This FVIIa/TF-initiated cascade is commonly referred to as the extrinsic pathway of blood coagulation. Although the extrinsic pathway contributes significantly to initial phases of thrombin generation, another pathway, is believed to play an essential role in maintaining the ability of the plasma to clot (1). This, the intrinsic pathway, is initiated by the activation of FXI to FXIa, and then proceeds through FIXa and FXa, and is also referred to as the maintenance pathway of blood coagulation. The 2 pathways converge at the activation of FIX and FX.

Not surprisingly, several inhibitory processes operate to prevent unchecked coagulation factor activity and total occlusion of the vasculature (2). For example, tis- sue factor pathway inhibitor (TFPI) downregulates FVIIa/TF activity through formation of a quaternary complex with FVIIa/TF and FXa (3), thus necessitating the presence of the intrinsic pathway, whereas activated protein $\mathrm{C}(\mathrm{aPC})$ serves directly as an anticoagulant by downregulating coagulant function via specific proteolysis of FV/FVa and FVIII/FVIIIa (4), 2 major cofactors required for the efficient formation of blood clots. In addition, aPC fulfills a profibrinolytic role by direct inactivation of other fibrinolytic inhibitors (5), and also serves in an indirect profibrinolytic capacity through quenching of thrombin, because loss of thrombin may lead to attenuation of the thrombin-catalyzed activation of a fibrinolytic inhibitor, TAFI (6). Furthermore, aPC has also been implicated in host-defense reactions that occur during intravascular inflammation (5).

Human FVII deficiency is a rare autosomal recessive disease, affecting approximately 1 in 500,000 individuals (7), that is caused by a variety of known mutations that decrease FVII antigen or catalytic levels. These patients often succumb to hemorrhagic diathesis. Conversely, PCdeficient patients are at increased risk for thromboembolic disorders, including neonatal purpura fulminans, disseminated intravascular coagulation, and recurrent venous thrombosis (8-10). No cases of total combined FVII/PC deficiency have appeared in the literature. 
Murine FVII, PC, and TFPI deficiencies have been generated through targeted total deletion of the FVII (11) and $P C(12)$ genes, and deletion of the first Kunitz domain of TFPI, which is responsible for TFPI/FVIIa interaction (13). FVII-deficient mice $\left(F \mathrm{VII}^{--}\right)$developed normally through embryogenesis, but succumbed to perinatal hemorrhage after birth (11). PC-deficient $\left(P C^{-1-}\right)$ embryos demonstrated a coagulopathic phenotype that resulted in their deaths immediately after birth (12). TFPI-Kunitz 1 domain-deficient mice $\left(T F P I^{\delta / \delta}\right)$ succumbed to coagulopathy with mortality from E9.5dpc to birth $(13,14)$. The latter 2 phenotypes underscore the importance of these anticoagulants during embryogenesis.

Generation of mice with combined deficiencies can provide significant insight into the pathology of individual gene deletion phenotypes and thus further delineate the functions of these proteins. A previous study demonstrated that with deficiency of FVII, the coagulopathy observed in the TFPI $I^{\delta / \delta}$ embryos was ameliorated, verifying the cause of death in these embryos to be solely dependent on its anticoagulant function (14). In the present case, we hypothesized that if the early mortality associated with PC deficiency in neonates was solely related to a hypercoagulable state, then removal of the primary initiation event of coagulation, namely FVII, could be sufficient to rescue the observed coagulopathy in $\mathrm{PC}^{-/-}$embryos and neonates. Any rescue of the $\mathrm{PC}^{-/-}$ neonates would then result in a phenotype similar to the bleeding tendencies observed in the $\mathrm{FVII}^{-/}$mice. Conversely, if an additional deficiency in FVII did not rescue the $P C^{-/-}$phenotype, the inference would be that either the early mortality observed in $\mathrm{PC}^{-/-}$neonates was not entirely due to a heightened coagulative state, or that in the absence of FVII, the functioning of the intrinsic pathway or other novel fibrin-generating processes is observed. To test these hypotheses, $\mathrm{FVII}^{+/-} / \mathrm{PC}^{+/-}$mice were generated and crossbred to provide $\mathrm{FVII}^{-/-} / \mathrm{PC}^{-/-}$ animals. The phenotypic analysis of progeny resulting from this breeding is the subject of the current report.

\section{Methods}

Animals. The generation of mice heterozygous for the FVII (11) and PC genes (12) has been described. FVII gene deletion results in replacement of exons 2-8 of the FVII gene with a neomycin phosphotransferase (neo) gene, thus eliminating its entire coding region. Targeted replacement of the $P C$ allele results in the deletion of all translatable exons, by substitution of exons 2-9 of the $P C$ gene with the neo gene. Both transgenic lines were backcrossed to the $\mathrm{F}_{4}$ generation in C57BL/6 mice ( $\sim 94 \%$ C57BL/6 background). Mice doubly heterozygous for FVII and PC $\left(\mathrm{FVII}^{+/-} / \mathrm{PC}^{+/-}\right)$, which were then bred to generate the animals studied herein, were generated by crossing these $\mathrm{F}_{4}$ generation $\mathrm{FVII}^{+/-}$and $\mathrm{PC}^{+/-}$mice.

Genotypic analysis. Tail tips, or portions of embryonic tissue or yolk sac, were isolated for the extraction of genomic DNA, as described previously (15). A 1.2-kb probe used for FVII genotypic analysis was isolated from digestion of the FVII targeting vector (pND.FVII) (11) with EcoRI and HindIII. A $2.2-\mathrm{kb}$ probe used for PC genotypic analysis was isolated from digestion of plasmid 130.3A1 (16) with BamHI and NotI. All probes were randomly labeled with $\alpha-\left[{ }^{32} \mathrm{P}\right] \mathrm{dCTP}$ (Amersham Life Sciences, Inc., Arlington Heights, Illinois, USA) using an Ambion Decaprime II (Ambion Inc., Austin, Texas, USA) random labeling kit. After digestion of genomic DNA with EcoRI and Southern hybridization with the appropriate probe, a 10.5 -kb band (wild-type FVII allele) or a $6.1-\mathrm{kb}$ band (recombined FVII allele) was observed for FVII genotyping, and an 11.5-kb band (wild-type PC allele) or a $7.5-\mathrm{kb}$ band (recombined $P C$ allele) was observed for the $P C$ genotyping.

Timed matings. Females were placed with nonsibling males in the late afternoon, and the morning of vaginal plug detection was assigned E0.5dpc. Pregnant females were euthanized, and the embryos were harvested at E12.5dpc, E14.5dpc, and E17.5dpc. Yolk sacs from early-stage embryos or tails of later-stage embryos were dissected for DNA isolation and genotyping.

Histology. Embryos were dissected free of maternal tissue. E12.5dpc and E14.5dpc embryos were fixed whole in freshly prepared $1 \%$ phosphate-buffered paraformaldehyde. Organs from E17.5dpc embryos were dissected and fixed similarly. Samples were dehydrated, embedded in paraffin, and sectioned at $4 \mu \mathrm{m}$ according to standard protocols. Sections were stained with hematoxylin and eosin (H\&E) to examine general tissue and cellular morphology, and with Masson's trichrome for detection of collagen (17). Immunostaining was also carried out on fixed tissues by direct immunoperoxidase or $\mathrm{ABC}$-immunoperoxidase (DAKO A/S, Copenhagen, Denmark) based staining, using commercial peroxidaselabeled murine primary antibodies, or indirectly, using peroxidase-labeled anti-IgG to label primary murine antigen-antibody complexes. Anti-fibrinogen antibody was purchased from Accurate Chemical and Scientific Corp. (Westbury, New York, USA). Anti-P-selectin, $\mathrm{CD} 45$, and Mac-3 antibodies were purchased from PharMingen (San Diego, CA). For immunostaining, epitope retrieval was accomplished by incubating sections in antigen retrieval buffer (DAKO A/S).

\section{Results}

Generation of $\mathrm{FVII}^{+/-} / \mathrm{PC}^{+/-}$mice and progeny from doubleheterozygous crosses. Mice carrying the $\mathrm{FVII}^{+/-}$genotype were crossed with those with the $P C^{+/-}$genotype to generate doubly heterozygous animals. Mice possessing the combined $\mathrm{FVII}^{+/-} / \mathrm{PC}^{+/-}$genotype appeared healthy and bred normally.

Nonsibling $\mathrm{FVII}^{+/-} / \mathrm{PC}^{+/-}$mice were crossed to generate progeny with the 9 genotypes possible from a double-heterozygous cross (Table 1). Postnatal genotypic analysis of these litters $(n=108)$ indicated that $\mathrm{FVII}^{-/-} \mathrm{PC}^{+/+}$and $\mathrm{FVII}^{-/-} / \mathrm{PC}^{+/-}$mice succumbed to the same intra-abdominal and intracranial hemorrhage that has been observed in $\mathrm{FVII}^{-/-}$mice (11). Similarly, $\mathrm{FVII}^{++/} / \mathrm{PC}^{-/-}$(6 observed, 9 expected $)$ and $\mathrm{FVII}^{+/-} / \mathrm{PC}^{-/-}$ 
Table 1

Distribution of postnatal and embryonic FVII/ PC genotypes derived from cross-matings of $\mathrm{FVII}^{+/-} / \mathrm{PC}^{+/-}$mice

\begin{tabular}{|c|c|c|c|c|c|c|c|c|c|}
\hline & $\begin{array}{c}\mathrm{FVII}^{+/+} / \\
\mathrm{PC}^{+/+}\end{array}$ & $\begin{array}{c}\mathrm{FVII}^{+/+} / \\
\mathrm{PC}^{+/-}\end{array}$ & $\begin{array}{c}\mathrm{FVII}^{+/-} / \\
\mathrm{PC}^{+/+}\end{array}$ & $\begin{array}{c}\mathrm{FVII}^{+/-} / \\
\mathrm{PC}^{+/-}\end{array}$ & $\begin{array}{c}\mathrm{FVII}^{+/+} / \\
\mathrm{PC}^{-/-}\end{array}$ & $\begin{array}{c}\mathrm{FVII}^{+/-} / \\
\mathrm{PC}^{-/-}\end{array}$ & $\begin{array}{c}\mathrm{FVII}^{-/-} / \\
\mathrm{PC}^{-/-}\end{array}$ & $\begin{array}{c}\mathrm{FVII}^{-/-} / \\
\mathrm{PC}^{+/-}\end{array}$ & $\begin{array}{c}\mathrm{FVII}^{-/-} \\
\mathrm{PC}^{+/+}\end{array}$ \\
\hline Postnatal & $6 / 9$ & $20 / 17$ & $19 / 17$ & $28 / 34$ & $6 / 9$ & $8 / 16$ & $0 / 8$ & $12 / 16$ & $9 / 9$ \\
\hline $\mathrm{E} 17.5^{\mathrm{A}}$ & $3 / 6$ & $9 / 12$ & $18 / 12$ & $22 / 23$ & $4 / 6$ & $15 / 12$ & $5 / 6$ & $9 / 12$ & $9 / 6$ \\
\hline E14. $5^{\mathrm{A}}$ & $3 / 4$ & $2 / 8$ & $14 / 8$ & $17 / 16$ & $3 / 4$ & $7 / 8$ & $5 / 4$ & $4 / 8$ & $4 / 4$ \\
\hline $\mathrm{E} 12.5^{\mathrm{A}}$ & $5 / 5$ & $16 / 10$ & $8 / 10$ & $15 / 20$ & $5 / 5$ & $15 / 10$ & $13 / 10$ & $13 / 10$ & $5 / 5$ \\
\hline
\end{tabular}

AThe number of animals (exhibiting/expected [\%]) of the indicated genotype out of a total of 137 postnatal, 94 E17.5 embryos, 64 E14.5 embryos, and 80 E12.5

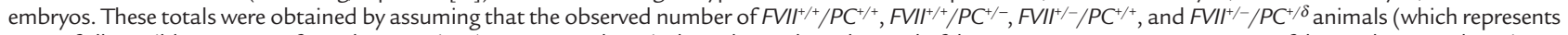
$9 / 16$ of all possible genotypes from these matings) were normal survival numbers. Thus, the total of these 4 genotypes represents $16 / 9$ of the total expected survivors.

(8 observed, 16 expected) mice were born, but succumbed to the same consumptive coagulopathy observed in the $\mathrm{PC}^{-/-}$mice (12). Thus, the increased C57BL/6 genetic contribution $(94 \%$ vs. $50 \%$ in both original studies), did not alter the phenotype of these $\mathrm{F}_{4}$ animals. However, no $\mathrm{FVII}^{-1-} / \mathrm{PC}^{-1-}$ neonates were identified ( 0 observed, 8 expected), possibly indicating that the combined FVII/PC deficiency resulted in embryonic lethality not previously observed in either of the FVII or PC single deficiencies. In addition, it was not conclusive whether a $50 \%$ level of FVII altered the survival or phenotype of the PC-deficient neonates. Thus, timed matings were initiated to harvest embryos at specific ages (Table 1).

Characterization of E17.5dpc embryos from double-heterozygous matings. Contrary to the postnatal genotypic analysis, at E17.5dpc, $F V I I^{-/} / \mathrm{PC}^{-/-}$embryos were present at their expected Mendelian frequency (5/93) at E17.5dpc (Table 1). However, initial examination of these embryos demonstrated a phenotype that had clearly not been observed in either of the single FVII or $P C$ gene deficiencies. Four of these 5 embryos were alive, but demonstrated severe edema, anemia, subcutaneous bleeding at the joints, and peripheral bleeding, as well as necrosis around the nasal area (Figure 1a). Although blood was also observed between the yolk sac and the embryo, yolk sac development and blood flow was normal (data not shown). In addition, placentas did not demonstrate abnormal development or hemorrhage. Unfortunately, the extensive hemorrhaging in the embryo prevented the collection of blood by direct cardiac puncture. Upon closer examination of the organs, fibrotic patches were observed in the liver, the brain demonstrated variable bleeding and necrosis, the ventricles were anemic, and the atria were clotted. These characteristics were also observed in the $P C$ singly deficient littermates. In 1 case, bleeding was also evident in the stomach and intestinal tract. H\&E staining verified the patches of liver fibrosis, encompassing red blood cell extravasation in the interstitia (Figure 1b). Interstitial fibrin deposition was also clearly evident in areas surrounding these fibrotic lesions (Figure 1c). Brain sections that were similarly stained demonstrated variable necrosis, massive hemorrhage, and fibrin deposition (Figure 1, d and e). Staining for collagen was positive in these areas, indicating the invasion of scar tissue, probably designed to contain hemorrhage and tissue damage (Figure 1f). Additionally, nonmacrophage $\mathrm{CD}^{2} 5^{+}$leukocytes were located in these areas of nonvascular fibrin deposition (Figure 1g). Similar to the observations in the brain, macroscopic characteristics of the atria were confirmed with $\mathrm{H} \& \mathrm{E}$ and antifibrin immunostaining. Abnormal hemorrhage and fibrin deposition was observed, as was the presence of abnormal collagen invasion in the internal atrial tissue (Figure 1, h-j). Areas of extravascular fibrin deposition did not stain positively for P-selectin-positive platelets (data not shown). The liver, brain, and atria of $\mathrm{FVII}^{+/+} / \mathrm{PC}^{-/-}$and $\mathrm{FVII}^{+/-} / \mathrm{PC}^{-/-}$mice, but not the wildtype or $\mathrm{FVII}^{-/-} / \mathrm{PC}^{+/+}$littermates, also demonstrated similar hemorrhage, fibrin deposition, and inflammatory cell infiltration as the $\mathrm{FVII}^{-1-} / \mathrm{PC}^{-/-}$embryos at this age (data not shown). Although the $\mathrm{FVII}^{+/-} / \mathrm{PC}^{-/-}$ embryos demonstrated similar organ coagulopathy, they did not present the severe edema and peripheral bleeding observed in the $\mathrm{FVII}^{-1-} / \mathrm{PC}^{-/}$embryos. Thus, it appears that a 50\% level of FVII does not significantly alter the coagulopathy observed in PC-deficient embryos. Additionally, these embryos were present at their expected Mendelian frequency, suggesting that their underrepresentation postnatally (Table 1) was due to our inability to identify these neonates before their death and consumption by their parents.

Characterization of E14.5dpc embryos from double-heterozygous matings. The progression of the coagulopathy in $\mathrm{FVII}^{-/-} \mathrm{PC}^{-/-}$embryos could be observed as early as E14.5dpc. Although $\mathrm{PC}^{-/-}$embryos appeared relatively normal with minimal bleeding anomalies, all $\mathrm{FVII}^{-/-} / \mathrm{PC}^{-/-}$embryos (5 out of 59 total embryos) exhibited peripheral and internal bleeding, with concomitant anemia (Figure 2a). Yolk sac development and blood flow generally appeared normal; however, $1 \mathrm{FVII}^{-/-} / \mathrm{PC}^{-/-}$embryo demonstrated blood pools within the amniotic sac. Extensive peripheral bleeding and fibrin deposition were confirmed histologically (Figure 2, b and c). Fibrin deposition, hemorrhage, and tissue degradation was also observed in the brain (Figure 2d), along the spine (Figure 2e), and in the liver. As with the E17.5 dpc FVII $I^{-/-} / \mathrm{PC}^{-/-}$ embryos, blood could not be collected by intracardiac puncture due to massive hemorrhaging. Of the 7 $\mathrm{FVII}^{+/-} / \mathrm{PC}^{-/-}$embryos examined at this age, 2 demon- 
a
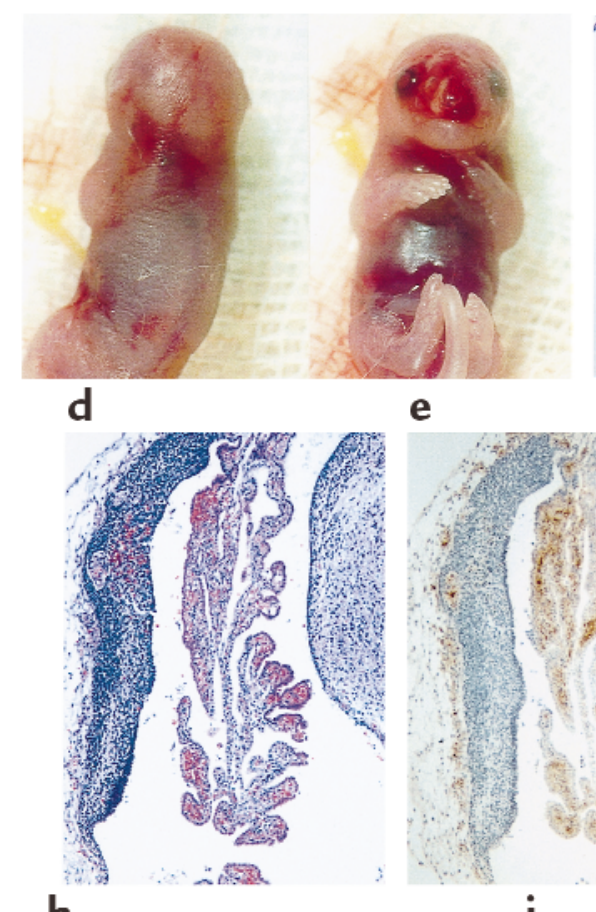

h

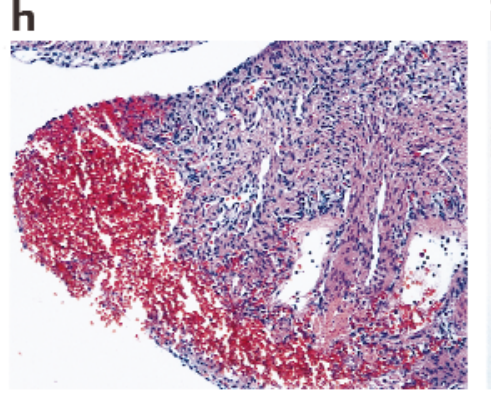

b

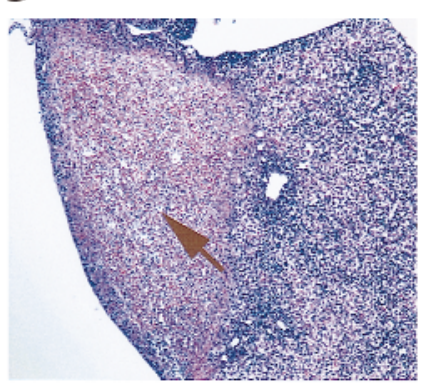

f

(1)

i c

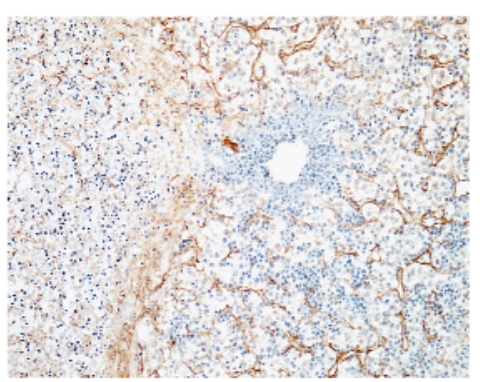

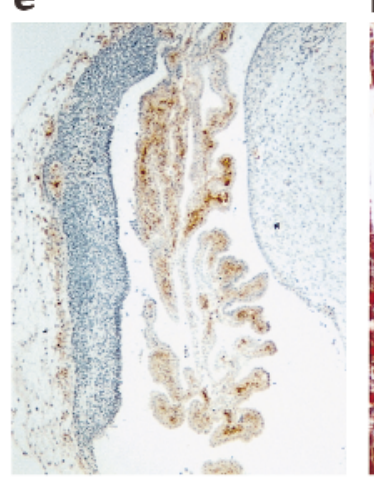

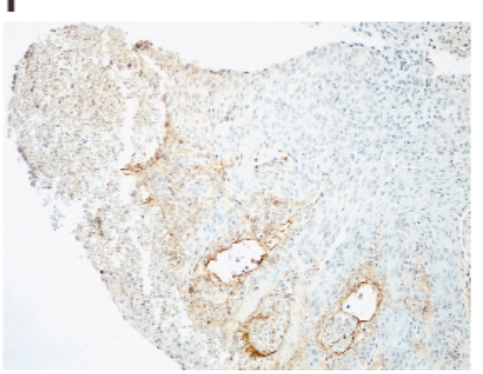

$\mathbf{g}$

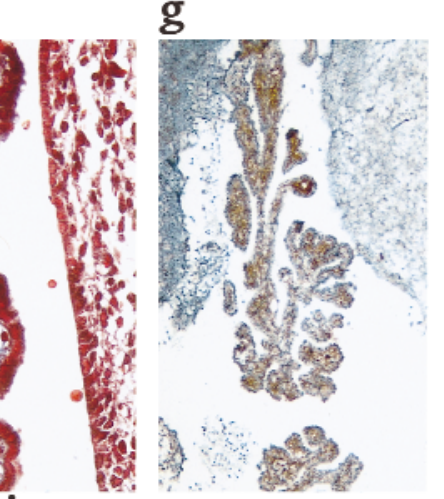

Figure 1

Analysis of an E17.5dpc FVII-/-/PC-/- embryo. (a) Gross appearance displaying significant internal and peripheral hemorrhage and edema. (b-c) H\&E staining of liver sections indicating areas of fibrosis within 1 of the lobes (arrow) (b); anti-fibrinogen immunostaining of parallel liver sections demonstrating interstitial fibrin deposition (brown) around these fibrotic patches (c). (d-g) Histological analyses of hindbrain sections indicating areas of hemorrhage (d), fibrin deposition (e), collagen deposition (blue) (f), and the presence of CD45-positive leukocytes (brown) (g). (h-j) Histological analyses of atrial sections confirming the presence of blood in the atria (h), fibrin deposition (i), and internal collagen invasion around these areas of fibrin (j).

strated variable bleeding spots on the skin and blood pools within the amniotic sac. Interstitial fibrin deposition was observed in the livers, and to a lesser extent, in the brains of these embryos, but there was no evidence of the peripheral or organ hemorrhage that was observed in the $\mathrm{FVII}^{-1-} / \mathrm{PC}^{-/-}$embryos (data not shown).

Characterization of E12.5dpc embryos from double-heterozygous matings. In contrast to the $\mathrm{E} 14.5 \mathrm{dpc}$ and E17.5dpc time points, E12.5dpc $F V I^{-/} / \mathrm{PC}^{-/}$embryos appeared developmentally normal, with no obvious external bleeding anomalies. Histologically, however, fibrin deposition, in the absence of accompanying hemorrhage, was observed in the liver interstitia and in vessels in the brain, neural canal, caudal artery, pulmonary cavity, dorsal aorta, and left hind-limb bud (Figure 3, a and b). $\mathrm{PC}^{-1-}$ embryos also had fibrin deposition in the liver interstitia and in vessels in the brain (data not shown). Again, areas of intra- and extravascular fibrin deposition did not stain positively for Pselectin-positive activated platelets (data not shown).

\section{Discussion}

It is generally accepted that thrombin generation and subsequent fibrin clot formation after vascular injury is a result of specific proteolytic events stemming solely from the combined efforts of the FVIIa/TF-initiated extrinsic pathway and the FXIa-initiated intrinsic pathway. Each of these pathways is controlled by specific inhibitors. TFPI attenuates the extrinsic pathway after the initial activation of small amounts of FX to FXa. Antithrombin III (ATIII) and aPC influence the intrinsic and extrinsic pathways through different mechanisms, by inactivating FXa and thrombin (ATIII), and FVa and FVIIIa (aPC). The importance of both TFPI and $\mathrm{aPC}$ is underscored by the fact that mice deficient in 
either TFPI or PC succumb to embryonic coagulopathy resulting in variable survival (12-14). These results are not altogether surprising considering that no TFPI-deficient patients have been identified, and that extensive (and often lethal) thrombotic events are observed in humans with homozygotic or compound heterozygotic PC deficiency. Additionally, PC has been regarded as a link between coagulation and inflammation (18). Although increased neutrophil infiltration was observed in several of the $\mathrm{PC}^{-/-}$embryos at E18.5dpc (12), an inflammatory-related cause of death was not thoroughly investigated.

In a previous study, complete loss of the FVII gene $\left(\mathrm{FVII}^{-/}\right)$resulted in rescue of the embryonic lethality in TFPI-deficient mice, demonstrating that the cause of death in these original TFPI-compromised embryos was due to uncontrolled FVII activity (14). Accordingly, to further understand the cause of death in PC-deficient mice and to attempt to delineate the roles of PC in vivo, $\mathrm{FVII}^{+/-} / \mathrm{PC}^{+/-}$mice were generated and intercrossed to produce doubly deficient progeny. However, in contrast to the FVII/TFPI double gene deletion study, postnatal genotypic analysis of litters produced from $\mathrm{FVII}^{+/-} / \mathrm{PC}^{+/-}$crosses did not indicate the presence of $\mathrm{FVII}^{-1-} / \mathrm{PC}^{-/-}$animals, suggesting that combined complete $F V I I / P C$ gene deficiency is not compatible with neonatal survival. Indeed, harvesting of embryos between E12.5 and E17.5dpc, sired from double-heterozygous crosses, indicated that $\mathrm{FVII}^{-1-} / \mathrm{PC}^{-/-}$ embryos, although alive and present at their expected Mendelian distribution, presented with an enhanced coagulopathic phenotype not observed in $P C$-deficient embryos, resulting in elevated peripheral hemorrhage and necrosis of external tissues. Thus, their significant postnatal underrepresentation was due to their immediate death either during or immediately after birth and their ultimate consumption by the parents, a phenomenon observed previously in FVII and PC singly deficient neonates $(11,12)$. Histologically, development of intra- and extravascular fibrin deposition began as early as E12.5dpc, which was not observed in the control littermate $P C^{-1-}$ embryos. This coagulopathy resulted in severe extravascular fibrin deposition in the liver, as well as atrial and brain tissue, with concomitant hemorrhage, as seen in the older $\mathrm{FVII}^{-1-}$ / $\mathrm{PC}^{-1-}$ embryos. P-selectin-positive immunostaining for the detection of activated platelets was not observed in fibrin-positive occluded vessels at E12.5dpc, nor in areas of extravascular fibrin deposition in later embryogenesis. CD45-positive leukocytes, although not identified as activated macrophages, were located intertwined in areas staining positively with fibrin
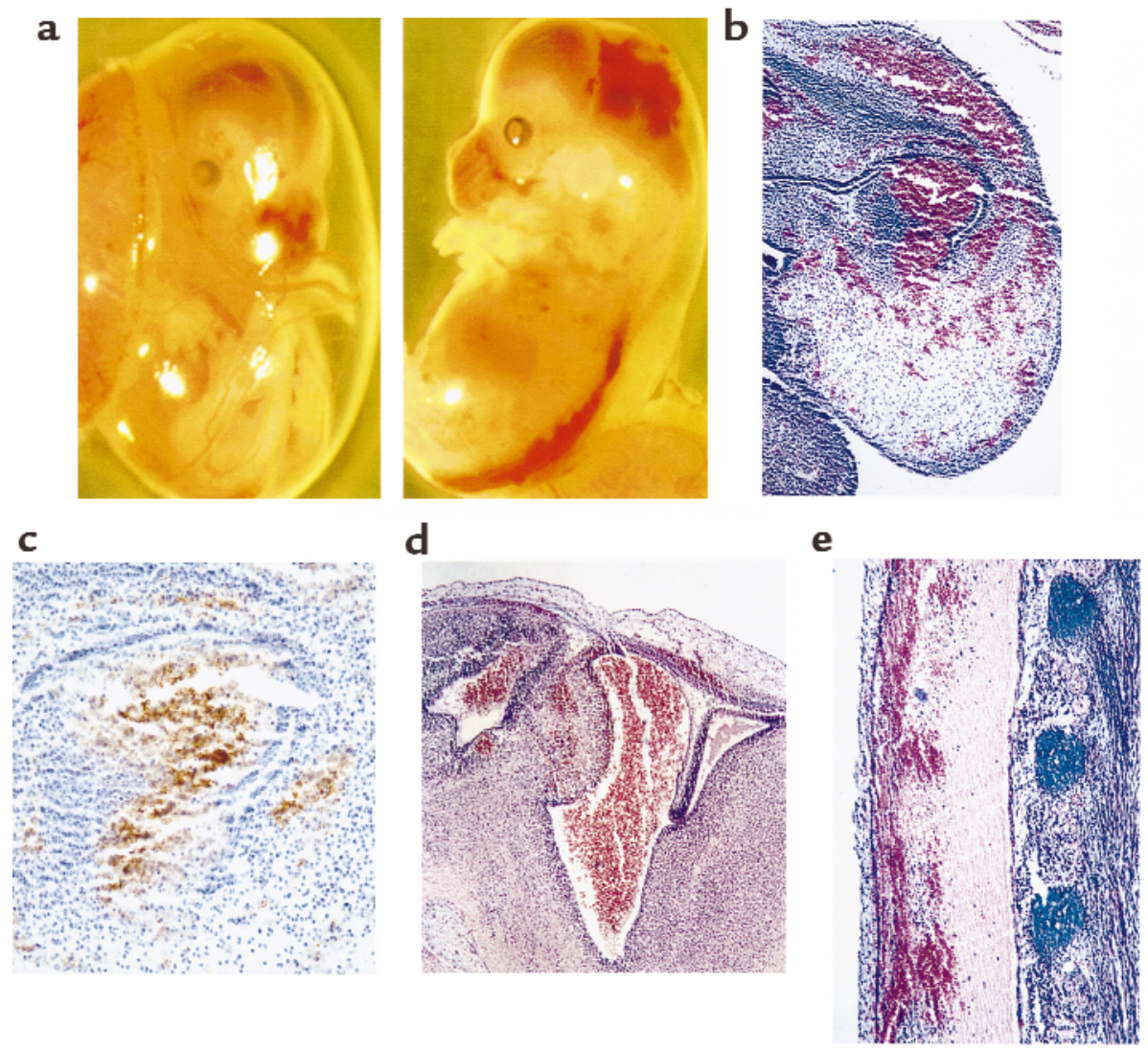

\section{Figure 2}

Analysis of an E14.5dpc FVII-/-/PC-/- embryo. (a) Gross appearance of an embryo. The hemorrhagic phenotype observed at E17.5dpc is readily occurring at E14.5dpc. Bleeding can be observed along the spinal column, in the brain, and in peripheral regions such as the snout. (b-e) Histological analyses of whole sectioned embryos. Hemorrhage (b) and fibrin deposition (c) are seen in the snout, plus bleeding in the lateral ventricle (d) and behind the spinal column (e). 


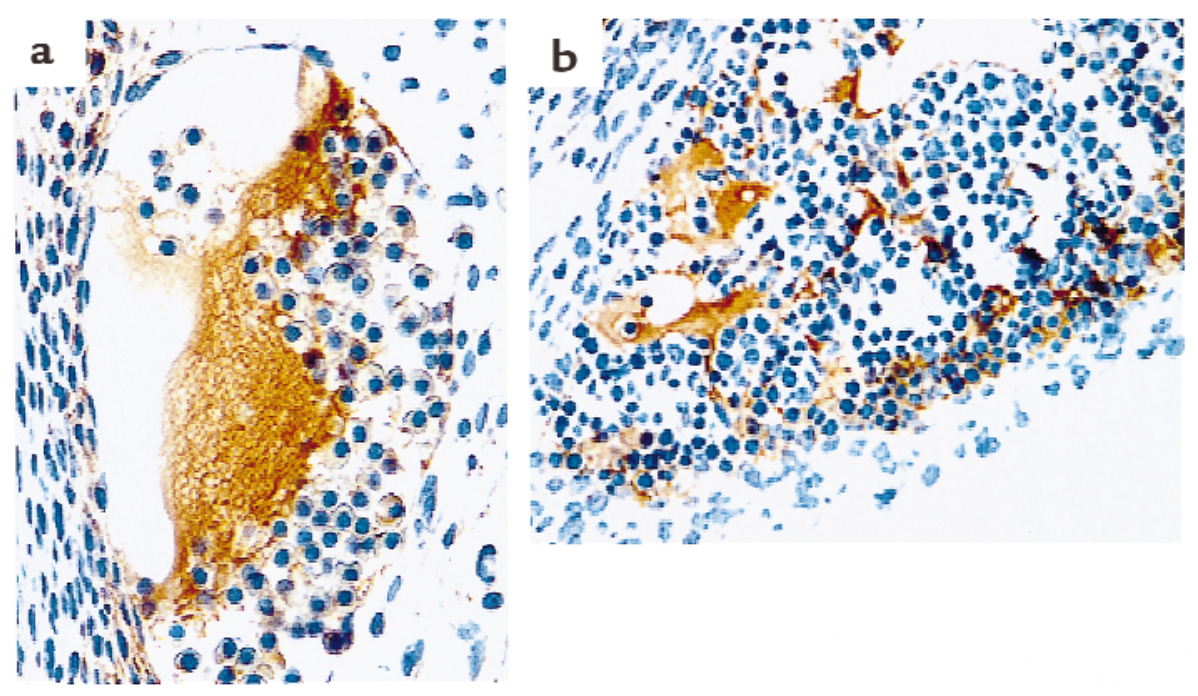

Figure 3

Anti-fibrinogen immunostaining of E12.5dpc FVII-/- $/ P C^{-/-}$embryo sections revealed fibrin deposition in brain vessels (a) and liver interstitia (b), characteristics not observed at these early embryonic stages in $\mathrm{PC}^{-/-}$embryos.

matrices in $\mathrm{FVII}^{-/-} / \mathrm{PC}^{-/-}, \mathrm{FVII}^{+/-} / \mathrm{PC}^{-/-}$, and $\mathrm{PC}^{-/-}$ embryos. Coagulopathy and hemorrhage were also observed, but without peripheral hemorrhage and edema, in $\mathrm{FVII}^{+/-} / \mathrm{PC}^{-/-}$littermates, suggesting a FVII gene dosage effect.

The fact that fibrin deposition was observed in the complete absence of FVII and PC was surprising. Our initial expectation was that the absence of fetal FVII would result in the loss of the initial burst of thrombin generation required to activate the FXI-initiated intrinsic pathway. Furthermore, the fact that a more severe coagulopathy was observed in PC-deficient embryos, in the absence of fetal FVII, was also unexpected. It is unlikely that the increased coagulopathy in the $\mathrm{FVII}^{-/-} / \mathrm{PC}^{-/-}$embryos and neonates was due to the higher C57BL/ 6 genetic contribution ( $94 \%$ vs. $50 \%$ in the original studies), because the phenotypes of the single knockout littermates did not differ from those with a $50 \%$ C57BL/ 6 background studied previously $(11,12)$. Instead, a plausible explanation for this heightened coagulopathy in the $\mathrm{FVII}^{-/-} / \mathrm{PC}^{-/-}$embryos may be twofold. First, in our model, the absence of the FVII gene may result in the loss of the anticoagulant function of the TFPI inhibitory complex, which requires FVIIa to function (14). Although in vitro studies have demonstrated that TFPI can bind to and inhibit FXa activity in the absence of FVIIa and TF (19), a mutant of TFPI lacking its Kunitz 1 domain (the FVIIa binding domain), but still containing its Kunitz 2 domain (the FXa binding domain), could not prevent coagulopathy in vivo (13). Thus, the functional redundancy afforded by the FXI-initiated intrinsic pathway, previously known as important in the maintenance of thrombin generation, is no longer controlled by either PC (which has been deleted) or TFPI (which is functionally irrelevant). Without this control, levels of FXa and thrombin are generated that can result in excessive amounts of fibrin formation. However, these amounts of thrombin and fibrin are lower due to the absence of FVII. In essence, the
FVII/PC double deficiency appears as a less severe case of TFPI deficiency. Perhaps not coincidental is that the $\mathrm{FVII}^{-/-} / \mathrm{PC}^{-/-}$embryos at E14.5dpc demonstrated massive fibrin deposition, hemorrhage, and necrosis similar that in the few TFPI ${ }^{\delta / \delta}$ embryos that survive to this age $(13,14)$. Secondly, increased levels of thrombin, in the absence of PC and TFPI, may result in elevated activation of TAFI, which would serve to downregulate plasmin fibrinolytic activity, tipping the hemostatic balance even further toward an enhanced prothrombotic state.

Thirdly, placental transfer of maternal FVII may account for the observed coagulopathy. However, a direct examination of maternal FVII levels in embryos failed to reveal such transfer, except when supraphysiological levels of maternal FVII were present $(11,20)$. Additionally, the rescue of the intrauterine lethality associated with a TFPI deficiency by the additional loss of FVII suggests that any possible level of maternal FVII is insufficient to cause coagulopathy in these embryos (14). Furthermore, if maternal FVII accounts for the total FVII in $\mathrm{FVII}^{-/-} / \mathrm{PC}^{-/-}$embryos, then their enhanced coagulopathic phenotype, as compared with their $\mathrm{PC}^{-/-}$ littermates, would not be observed. Taken together, although very small (and as yet undetectable by currently available assays) amounts of maternal FVII in embryos could influence other functions of TF, it is highly unlikely that maternal FVII plays a role in the observed combined $\mathrm{FVII}^{-/-} / \mathrm{PC}^{-/-}$coagulopathy.

A fourth possible explanation for the incomplete rescue of the $\mathrm{PC}^{-/}$genotype by the additional loss of FVII is that other novel fibrin-generating mechanisms exist outside of the FXI-initiated intrinsic pathway. Further combined deficiency models will be invaluable in addressing this topic in an in vivo setting.

A final intriguing issue is that activation of the intrinsic pathway appears to be occurring in the absence of FVIIa/TF activity, which has been believed to be the initiator of low-level thrombin generation required to activate FXI and the intrinsic maintenance pathway. 
The presence of fibrin in $\mathrm{FVII}^{-/-} / \mathrm{PC}^{-/-}$embryos raises the issue of the mechanism of FXI activation in the absence of FVII, if initial thrombin levels are generated through this route, and suggests that other mechanisms are in place to activate FXI in vivo.

\section{Acknowledgments}

The authors thank Mayra Sandoval-Cooper for technical assistance. These studies were supported by grant HL-19982 (to F.J. Castellino) and HL-63682 (to V.A. Ploplis) from the National Institutes of Health, a grant from the W.M. Keck Foundation (to F.J. Castellino), and by the Kleiderer/Pezold Family Endowed Professorship (to F.J. Castellino).

1. Broze, G.J. 1995. Tissue factor pathway inhibitor and the revised theory of coagulation. Annu. Rev. Med. 46:103-112.

2. Broze, G.J., Likert, K., and Higuchi, D. 1993. Inhibition of factor VIIa-tissue factor by antithrombin III and tissue factor pathway inhibitor. Blood. 82:1679-1680.

3. Broze, G.J., et al. 1988. The lipoprotein-associated coagulation inhibitor that inhibits the factor VII-tissue factor complex also inhibits factor Xa: insight into its possible mechanism of action. Blood. 71:335-343.

4. Kisiel, W., Canfield, W.M., Ericsson, L.H., and Davie, E.W. 1977. Anticoagulant properties of bovine plasma protein $\mathrm{C}$ following activation by thrombin. Biochemistry. 16:5824-5831.

5. Taylor, F.B., et al. 1987. Protein C prevents the coagulopathic and lethal effects of Escherichia coli infusion in the baboon. J. Clin. Invest. 79:918-925.

6. Bajzar, L., Morser, J., and Nesheim, M. 1996. TAFI, or plasma procarboxypeptidase B, couples the coagulation and fibrinolytic cascades through the thrombin-thrombomodulin complex. J. Biol. Chem. 271:16603-16608.

7. Fadel, H.E., and Krauss, J.S. 1989. Factor VII deficiency and pregnancy. Obstet. Gynecol. 73:453-454.

8. Tuddenham, E.G.D., et al. 1989. Homozygous protein C deficiency with delayed onset of symptoms at 7 to 10 months. Thromb. Res. 53:475-484.

9. Marlar, R.A., and Neumann, A. 1990. Neonatal purpura fulminans due to homozygous protein $\mathrm{C}$ or protein $\mathrm{S}$ deficiencies. Semin. Thromb. Hemost. 16:299-309.

10. Tuddenham, E.G., Pemberton, S., and Cooper, D.N. 1995. Inherited factor VII deficiency: genetics and molecular pathology. Thromb. Haemost. 74:313-321.

11. Rosen, E., et al. 1997. Mice lacking factor VII develop normally but suffer fatal perinatal bleeding. Nature. 390:290-294.

12. Jalbert, L.R., et al. 1998. Inactivation of the gene for anticoagulant protein $\mathrm{C}$ causes lethal perinatal consumptive coagulopathy in mice. J. Clin. Invest. 102:1481-1488.

13. Huang, Z.-F., Higuchi, D., Lasky, N., and Broze, G.J. 1997. Tissue factor pathway inhibitor gene disruption produces intrauterine lethality in mice. Blood. 90:944-951.

14. Chan, J.C.Y., et al. 1999. Factor VII deficiency rescues the intrauterine lethality in mice associated with a tissue factor pathway inhibitor deficit. J. Clin. Invest. 103:475-482.

15. Carmeliet, P., et al. 1994. Physiological consequences of loss of plasminogen activator gene function in mice. Nature. 368:419-424.

16. Jalbert, L.R., et al. 1998. Nucleotide structure and characterization of the murine gene encoding anticoagulant protein C. Thromb. Haemost. 79:310-316.

17. Masson, P. 1929. Trichrome stainings and their preliminary technique. J. Tech. Meth. 12:75-90.

18. Esmon, C.T., Taylor, F.B., and Snow, T.R. 1991. Inflammation and coagulation: linked processes potentially regulated through a common pathway mediated by protein C. Thromb. Haemost. 66:160-165.

19. Huang, Z.F., Wun, T.C., and Broze, G.J. 1993. Kinetics of factor Xa inhibition by tissue factor pathway inhibitor. J. Biol. Chem. 268:26950-26955.

20. Beeby, T.L., Chasseaud, L.F., Taylor, T., and Thomsen, M.K. 1993. Distribution of the recombinant coagulation factor ${ }^{125} \mathrm{I}$-rVIIa in rats. Thromb. Haemost. 70:465-468. 